\title{
El significado y la evolución del término «coeducación» con el cambio de siglo: el caso de los centros escolares de Vitoria-Gasteiz
}

The meaning and evolution of the term "coeducation" with the turn of the century: the case of the schools of Vitoria-Gasteiz

\author{
Amaia Alvarez Uria \\ e-mail: amaia.au@ehu.eus \\ Euskal Herriko Unibertsitatea. España
}

María Teresa Vizcarra Morales

e-mail: mariate.bizkarra@ehu.eus

Euskal Herriko Unibertsitatea. España

Gema Lasarte Leonet $\mathbb{D}$

e-mail: gema.lasarte@ehu.eus

Resumen

Euskal Herriko Unibertsitatea. España

El término coeducación se suele utilizar cuando se habla de perspectiva de género o feminista en educación o al situar este tema en la escuela. Sin embargo, parece que en ciertos ámbitos la coeducación es un tema del pasado, algo que ya se ha superado en educación. Por esta razón cuando se habla de ello hay personas que cuestiona la necesidad de tratar estos temas como parte del currículum, ya que creen que es solo la familia la que debe transmitir este tipo de valores, y sin embargo, hoy en día aún no se ha logrado llegar a una escuela coeducativa. Este estudio pretende comprender cómo se entiende el término coeducación y cuál ha sido su evolución. La presente investigación relata un estudio diagnóstico centrado en 30 centros escolares de VitoriaGasteiz, en el que a través de cuestionarios a profesorado y grupos de discusión con familias, profesorado y alumnado. Se trata de un estudio de caso que recoge diferentes concepciones sobre la coeducación en los centros de la ciudad. Los resultados aportados constatan que es necesaria una actualización conceptual, ya que este término sigue asociado a la escuela mixta y a un concepto de igualdad basado en un sistema binario, y no se relaciona con otras opciones más recientes relacionadas con la feminización del conocimiento, la revisión de la masculinidad o la visibilización y la valoración de la diversidad.

Palabras clave: coeducación; escuela mixta; igualdad de oportunidades; feminismo; diversidad cultural

\begin{abstract}
As we speak about gender perspective or feminism in education or at the school, people use the coeducation concept. In some areas it seems like coeducation was an ancient subject, an acquired matter in education. For this reason when it comes to talking about it there are people who question the need to treat these issues as part of the curriculum, since they believe that it is only the family that should transmit this type of values, and yet today they still do not have managed to reach a coeducational school. However, we have not reached a coeducative school yet. That is why this research pretends to know how this concept is understand and which has been its evolution. In the present article the reader will find a diagnosis made in 30 schools in VitoriaGasteiz. In those schools has been done focus groups with families, teachers and students, and we have found different ways of looking to this term, coeducation. It is a case study that gathers different conceptions about coeducation in the city. We have concluded that an update is needed for this concept, since it is still related to the mixed school (girls and boys studying together), and to the idea of equality based on a binary social system. Thus, it is not related to more recent ideas, as the feminization of knowledge, the revision of the hegemonic masculinity, or the visibilization and valuation of the diversity.
\end{abstract}

Keywords: coeducation; coeducational schools; equal opportunity; womens liberation movement; cultural diversity

Recibido / Received: 23-01-2019

Aceptado / Accepted: 23-04-2019

Publicación en línea / Published online: 01-07-2019

Cómo referenciar este artículo / How to reference this article:

Álvarez-Uria, A., Vizcarra Morales, M. T., \& Lasarte Leonet, G. (2019). El significado y la evolución del término «coeducación» con el cambio de siglo: el caso de los centros escolares de Vitoria-Gasteiz. Tendencias Pedagógicas, 34, pp. 62-75. doi: 10.15366/tp2019.34.006 


\title{
1. La coeducación y la evolución de su significado
}

La vida de las palabras, que evolucionan con el tiempo, es una muestra de que el lenguaje es cambiante y tiene un componente histórico. Es eso lo que ha sucedido con las palabras feminismo, género o coeducación, el término al que nos aproximamos en aras de hallar su significado y conocer la evolución del mismo. Por ello, se va a revisar cómo se ha definido en los últimos años.

La palabra coeducación ha vivido diferentes épocas y situaciones socio-históricas, por lo que ha ido evolucionando y ocasionalmente entre algunas personas de la comunidad escolar (mayoritariamente situadas lejos del feminismo) se siente como una palabra vieja, caduca, pasada de moda, lo que lleva a dejarla a un lado y no considerarla como una prioridad a trabajar hoy en día en las escuelas, a no ser que se tenga conciencia con el tema.

\begin{abstract}
«La demanda de coeducación, en nuestro país, es una constante desde el último tercio del siglo XIX y hoy seguimos hablando de coeducación. Lo que parece obligado preguntarnos es: ¿al decir coeducación hablamos de la misma demanda de hace un siglo?, ¿es que perviven los mismos problemas?, ¿es que se trata de una vieja fórmula aún inalcanzada?, ¿ंo tal vez esta constante aspiración nos está llevando a convertir en objetivo lo que tan sólo era un medio?, ¿no nos estará impidiendo pensar en otros medios, otras estrategias?» (Ballarín, 2001, p. 32)
\end{abstract}

La palabra coeducación ha recorrido un camino de dos siglos a nivel global y de alrededor de cuatro décadas a nivel local. En la cultura anglosajona occidental los primeros centros educativos mixtos, que aceptaban alumnas junto a los alumnos, datan de principios del siglo XIX. En el estado español, todo lo que pudo adelantarse a finales del siglo XIX y principios del siglo XX, en especial en la II República desaparece tras la Guerra Civil y no aparece hasta después de la muerte de Franco. «Hubo que esperar a la llegada del régimen democrático y de la escuela comprensiva para proponer que niños y niñas se educaran bajo los valores de la igualdad, lo que se conoce como escuela mixta» (Calvo, Susinos, y García, 2011, p. 566).

En el siglo XIX con la constitución de 1812 se consiguieron casi los mismos derechos educativos para niñas y para niños, aunque esta situación no duró demasiado (Araque, 2009). Hay que esperar hasta 1857 con la Ley Moyano para que se creen las Escuelas Normales de maestras, que tienen un currículum diferenciado y menos exigente con respecto a las escuelas de maestros (Arenas, 2006). A principios del siglo XX existen varias experiencias coeducativas, aunque no recibieron ese nombre, de manos de la Escuela Moderna, la Escuela Nueva, y la Institución Libre de Enseñanza (MartínezGorroño y Hernández-Álvarez, 2014). A comienzos del siglo XX, en 1909, la educación era obligatoria para niñas y para niños, aunque había un alto grado de absentismo escolar, pues compaginaban los estudios con el trabajo. La segunda República en 1931 promulga la escuela mixta y la hace obligatoria en 1937 (Pujadas, 2012). En dicho período se dieron avances y transformaciones profundas, se promulga la coeducación en las aulas, se intenta hacer frente a un alto nivel de analfabetismo y se reconoce a niñas y niños como sujetos educativos activos, que deben educarse en igualdad (Ianni, 2008). Sin embargo, todas estas iniciativas de la escuela mixta, se truncaron con la guerra civil y el franquismo, que supone una vuelta a la escuela segregada, donde las mujeres reciben una educación estereotipada, creando los colegios para señoritas ya la formación para el hogar dirigida por la Sección Femenina (Ballarín, 2006). Durante este período existen diferentes iniciativas a favor de la coeducación que no llegaron a fraguar (Codina, 2002)

A partir de la Ley general de 1970 de Villar Palasí, se promulga la educación mixta que no será obligatoria hasta el curso 1984/1985 y que traerá una igualdad formal al incluir a las chicas en las clases con los chicos, pero no una igualdad real, pues el currículo es masculino y deja atrás el currículum femenino (Codina, 2002). Es por esto, que la definición de «coeducación» primeramente se fija en la co-educación de mujeres y hombres, o de blancos y negros en los Estados Unidos de América en la década de 1960 (Riley, 2010). Es decir, el sinónimo de escuela mixta, y el antónimo de escuela segregada; una co-instrucción o co-enseñanza (Subirats, 1994). De ahí surge la confusión de que la educación mixta es una herramienta suficiente para la consecución de la igualdad entre hombres y mujeres (Suberviola, 2012).

Sin embargo, con el paso del tiempo, se vio que la escuela mixta, o la educación conjunta, no era suficiente desde un punto de vista emancipador y feminista. A partir de ese momento se incidió en la educación igualitaria, paritaria o equitativa, a la vez que se respetaban, valoraban y visibilizaban las 
diferencias de cada persona. Y así la palabra coeducación pasó a significar educación para la igualdad. Marian Moreno (2013) explicita que «coeducar» es sinónimo de «educar para la igualdad» y que no se debe confundir con «escuela mixta», pues no es lo mismo juntar a niños y niñas en un aula que educar para la igualdad. Ya que, como hemos mencionado, la escuela mixta posibilitó que los modelos fueran androcéntricos y excluyó la cultura de las mujeres del currículum y de los procedimientos (Moreno, 2013). También Yugeros (2015) identifica la coeducación con la igualdad obtenida gracias a la lucha de los grupos feministas.

En 1990 con la LOGSE se pasó de una igualdad formal conseguida por la educación mixta, a una mayor aproximación a la igualdad real, ya que el currículo incluye entre sus líneas transversales, la coeducación como contenido curricular. Se crearon materiales didácticos y se generó una profunda reflexión en los centros. En la Comunidad Autónoma Vasca esta reflexión va de la mano de Emakunde, el Instituto vasco de la mujer que fue creado en 1988 y celebraba todos los años jornadas coeducativas para el profesorado de los centros educativos no universitarios. En cualquier caso la propia ley recoge que el currículum:

«permite avanzar en la lucha contra la discriminación y la desigualdad, sean éstas por razones de nacimiento, raza, sexo, religión u opinión, tengan un origen familiar o social o se arrastren tradicionalmente (...) puede y debe convertirse en un elemento decisivo para la superación de los estereotipos sociales asimilados a la diferenciación por sexos, empezando por la propia construcción y uso del lenguaje» (LOGSE, 1990).

Desde la LOGSE se han dado pocos avances a favor de la coeducación, aún así, la Ley Orgánica de Educación de 2006 expresa que los fines de la educación persiguen:

«el pleno desarrollo de la personalidad y de las capacidades afectivas del alumnado, la formación en el respeto de los derechos y libertades fundamentales y de la igualdad efectiva de oportunidades entre hombres y mujeres, el reconocimiento de la diversidad afectivo-sexual, así como la valoración crítica de las desigualdades, que permita superar los comportamientos sexistas» (LOE, 2006).

En muchos ámbitos educativos, hoy en día, se entiende el concepto de coeducación como una educación no machista, no androcéntrica y no sexista, que supera estereotipos y roles de género, y trata de acabar con las desigualdades y discriminaciones inherentes al sistema patriarcal o a la ideología de género. Según podemos leer en la tesis de Heredero de Pedro (2013), se trata de educar al margen de la imposición social del género y potenciando el desarrollo individual. La Ley Orgánica de Mejoramiento de la Calidad Educativa (LOMCE, 2013), habla de prevenir la violencia de género y fomentar la igualdad entre hombre y mujeres, pero no parecen contenidos en el currículo, o que den un espacio a este tipo de contenidos (Subirats, 2017).

En las aulas habría que proponer tareas y actividades en dos direcciones: una, en la que las chicas continúen con la labor de superación de su rol de género, aumentando su cota de autonomía y libertad; y otra, haciendo una revisión crítica de la masculinidad hegemónica, algo que en estos momentos está lejos de ser una práctica común en las aulas.

Las investigaciones que han estudiado la igualdad de género en la escuela han generado replanteamientos de la planificación educativa, pero, pese a los logros cosechados en este ámbito se observan vacíos como la ausencia de análisis que identifique modelos alternativos a la masculinidad que permitan superar la violencia de género y el acoso en las generaciones más jóvenes en los centros educativos (Ríos, 2015). Aún prevalece una gran presión social para que los niños sigan el modelo de la masculinidad dominante:

«El modelo dominante de masculinidad ha evolucionado menos en los últimos años, que el de feminidad. Como todo modelo que confiere poder, es más difícil de poner en cuestión, de criticar, de modificar, puesto que su adopción es asimilada a las posiciones prestigiosas, mientras que los modelos de comportamiento que van simbólicamente unidos a posiciones dominadas o menos valoradas son más fáciles de rechazar, discutir, evaluar y modificar» (Subirats y Tomé, 2007, p. 62). 
Pero aún se ha dado un paso más al entender, desde un punto de vista pedagógico, que las personas deben tener libertad y oportunidades para poder desarrollarse integralmente. Por lo que el siguiente estadio es el del desarrollo personal y de la construcción identitaria con garantías de estar en un entorno seguro y con posibilidades de desmontar prejuicios y equilibrar las relaciones de dominación/sumisión para romper con la norma social imperante respecto al sexo/género. Este punto de vista holístico se plasma en la propuesta de pentacidad, como el desarrollo integral de capacidades y valores estructurados en cinco ámbitos: identidad, sociedad, mente, cuerpo y emociones (Salas, 2003). También pone el objetivo en «la consideración de la totalidad de las personas como "sujetos" y por la relación entre personas diferentes sin que esta diferencia comporte una desigualdad» (Heredero de Pedro, 2013, p. 58). O pensando la educación como un instrumento para la socialización de las personas que esculpe la personalidad y las formas de actuar y se transmiten los modelos genéricos (Subirats, 2016).

Un punto clave en esta dirección es la incorporación de los saberes femeninos en la educación. Se deber reconocer que ha habido dos culturas, la de los hombres, que se ha enseñado, aprendido y valorado positivamente en las escuelas y se sigue haciendo así. Y la de las mujeres, que ha sido invisibilizada, relegada al ámbito doméstico y denostada o valorada negativamente. Por lo que no se ha educado a la ciudadanía del futuro como seres autónomos, ya que se han preparado para el ámbito público, pero no para el privado. Según Solsona (2008, p. 227) «hoy tenemos que modificar la centralidad de los saberes y trabajos considerados tradicionalmente masculinos en la cultura escolar y crear una nueva en la que se valoren los sentimientos y las emociones, las capacidades relacionales, los saberes y las experiencias de las mujeres y los saberes asociados al cuidado».

Otro concepto que en los últimos años está ganando terreno es el de la diversidad. Hace tiempo que en educación se trata de atender a la diversidad que cada vez es más rica y visible en las aulas. Y, aunque al principio al hablar de este tema se referían a los diferentes ritmos, funcionalidades y capacidades de aprendizaje, por un lado, y a la multiculturalidad por otro, ahora está claro que hay muchas diversidades, y que entre ellas están las relativas al sexo, al género y a la sexualidad. Por ello la coeducación se empieza a relacionar con la superación del binarismo, la presencia de la transexualidad, o la descentralización de la heterosexualidad. En palabras de Mirabilia, por medio de la coeducación se consigue el reconocimiento y la valoración de las diferencias para poder realizarse y desarrollarse libremente, y también que las desigualdades se atenúen y que se consiga la igualdad en derechos y oportunidades (2011). Porque, según Cabeza (2006), se logra eliminar la jerarquización de un género sobre otro, y, en vez de educar a niños y niñas idénticamente basándose en un sistema de valores masculino sin tener en cuentas las diferencias entre los géneros, se pretende integrar, valorar y respetar la diversidad. Al fin y al cabo, «La igualdad de diferencias en términos de identidad de género implica incorporar la pluralidad como componente enriquecedor y no excluyente» (Ríos, 2015, p. 495).

Para finalizar este camino de conceptualización, evolución y cambio semántico del término coeducación, debemos nombrar otros tipos de educación que actualmente están en boga y que se relacionan directamente con la coeducación: la educación en valores, la educación emocional-afectiva, la educación para la ciudadanía, la educación para la paz, la escuela inclusiva, y las competencias para la vida. Todas ellas se refieren a diferentes puntos citados anteriormente como la perspectiva globalizadora e integral e integradora, la confluencia entre las culturas, experiencias y saberes masculinos y femeninos; y la aceptación y reconocimiento de la diversidad y de su riqueza. Por ejemplo, Leal y Arconada (2011) proponen un decálogo para una escuela coeducadora y pro-igualitaria, en la que aparecen entre otros aspectos la valoración de la «no-violencia», los cuidados, y la responsabilidad individual. En la misma línea, Cabeza apunta que se fomenten valores, actitudes y comportamientos éticos, a la vez que se desarrollan actitudes críticas ante los conflictos que afectan a la convivencia y los problemas de la sociedad (Cabeza, 2006).

En resumen, la coeducación se perfila hoy basándose en valores como democracia, solidaridad, o justicia social, con la finalidad de que gracias a la educación se consigan el desarrollo personal y la construcción común en las escuelas del siglo XXI. Así que, desde un punto de vista universal, según Romero y Lugo (2014, p. 1036), la perspectiva de género es necesaria para la consecución de una sociedad democrática y la superación de la vigente cultura androcéntrica, pues «un enfoque integrado precisa de un proceso de cambio a todos los niveles desde una puesta en marcha de nuevos procedimientos a nivel técnico como organizacional».

La escuela, en sí, reproduce la sociedad en la que está inscrita, y es por ello, que es de máxima importancia incidir en la formación del profesorado y en su preparación. De esta manera, se podrá acompañar y educar a la sociedad del futuro como personas capaces, completas y autónomas que 
convivan en un mundo complejo. Como hemos visto anteriormente, la escuela produce y reproduce estereotipos de género y fundamenta la construcción y socialización del sistema sexo-género. Esta institución, según de Tomé (2001), transmite conocimientos, normas, principios y valores. Todas las personas que forman parte de la comunidad escolar son responsables de la educación y de la convivencia en ella. Se deben cuidar la formación y los recursos de las personas que harán el acompañamiento en este viaje que es la educación (Mirabilia, 2011).

Siguiendo estas premisas en este segundo milenio en el estado español el Ministerio de Igualdad publica, entre otras, la Guía de coeducación del Instituto de la mujer (2008) en el que se propone una definición de coeducación, medios para identificar buenas prácticas y para revisar material didáctico, y recursos para trabajarlo en la escuela y hacer un seguimiento de lo realizado. He aquí la definición que proponen:

\begin{abstract}
«La propuesta pedagógica actual para dar respuesta a la reivindicación de la igualdad realizada por la teoría feminista, que propone una reformulación del modelo de transmisión del conocimiento y de las ideas desde una perspectiva de género en los espacios de socialización destinados a la formación y el aprendizaje» (2008, p. 17).
\end{abstract}

En el entorno de la Comunidad Autónoma Vasca surgen algunas iniciativas como el programa Nahiko que es un programa de tutorías para educación primaria de Emakunde (Instituto de Igualdad del Gobierno Vasco), que lleva aplicándose en los centros escolares desde 2003. Lo definen y presentan de la siguiente manera: «Nahiko es un programa coeducativo para la Igualdad, el respeto y la no-violencia. Se trata de un programa para la convivencia en igualdad, mediante la educación en valores, tomando como base los derechos humanos y el análisis de los roles de género». (Emakunde, 2003, p. web). Los objetivos de este plan son «Mejorar la convivencia y las relaciones entre alumnas y alumnos, educar para la igualdad y los Derechos Humanos y prevenir la violencia contra las mujeres». (Emakunde, 2003, p. 2)

Y para ello, propone materiales con el fin de desarrollar «nuevos modelos de convivencia» y posibilitar lo siguiente: «- Desarrollo y autonomía plena de cada chica y cada chico. - Responsabilidad en todos los ámbitos de la vida. - Construcción de relaciones sin violencia entre las personas. - Análisis y reflexión de la realidad y las relaciones desde una perspectiva de género. - Nuevas masculinidades sin violencia» (Emakunde, 2003).

En 2013, el Gobierno Vasco publica el plan director para la Coeducación y la prevención de la violencia de género en el sistema educativo que tiene como fin avanzar hacia una escuela coeducativa, y pone en marcha un programa piloto en el que participan 25 centros escolares. Este texto expresa que aunque se considera que las mujeres han demostrado su potencialidad en el sistema educativo, aún persisten en las relaciones entre chicos y chicas una asimetría de género que está pendiente de superar.

En marzo de 2019, el Gobierno Vasco celebra las VI jornadas del programa educativo Nahiko que con una trayectoria de más de 15 años mostraba el trabajo realizado en 42 centros escolares durante el período 2017-2019.

Junto con esa iniciativa del Gobierno Vasco, el Gobierno de Navarra ha ido más allá y acaba de presentar a principios del curso 2018/2019, después de unas jornadas y un curso de experiencia piloto, SKOLAE, su plan de igualdad previsto para el período 2017-2021. Así se presenta en su página web:

«SKOLAE pretende facilitar el aprendizaje de una competencia global que permita elegir a niños y niñas el proyecto vital propio desde la libertad y la diversidad de opciones, sin condicionantes de género, aprendiendo a identificar las desigualdades, a luchar contra ellas y a ejercer su derecho a la igualdad en el ámbito de su cultura, religión, clase social, situación funcional, orientación sexual, etc.» (Gobierno de Navarra, 2017, p. web)

Y para conseguirlo, el alumnado tendrá que realizar diversos itinerarios en los que aprenderá lo siguiente: «1. Crítica frente a la desigualdad y capacidad para el cambio; 2 . Autonomía, independencia personal y centralidad del empleo; 3 . Liderazgo, empoderamiento y participación social; 4. Sexualidad, convivencia y prevención de la violencia» (Gobierno de Navarra, 2018, p. 16)

Tanto la propuesta del Gobierno Vasco como la del de Navarra parten de una idea de igualdad para llegar a diferentes objetivos dentro del marco de coeducación. Pero, ¿es así como se percibe esta cuestión en la comunidad educativa? 
El objetivo de este artículo es comprender la realidad escolar en torno a la coeducación en los centros escolares, por ello, los objetivos son conocer cómo entienden la escuela coeducadora, qué características tiene y si su significado ha evolucionado o no. Así como, descubrir cómo se entiende el papel del profesorado en el camino hacia la coeducación, la percepción que se tiene en relación a su formación y las diferentes actitudes, sensibilidades y propuestas que aparecen en torno a este tema. Por ello, se recogen las variadas definiciones que los diferentes colectivos educativos tienen sobre el concepto de coeducación, es decir, ¿qué entienden las familias, el profesorado, los equipos directivos y el alumnado por coeducación?

\section{Método}

Este artículo es un estudio de caso que pretende hacer un diagnóstico evaluativo de la situación de la coeducación en los centros educativos de Vitoria-Gasteiz ( 0 a 18 años). Fue realizado en 30 centros educativos a instancias del departamento de Educación del Ayuntamiento de Vitoria-Gasteiz (País Vasco), realizado en 2013 y se finaliza en 2014, se buscaba conocer cómo era percibida la Coeducación en los centros educativos. Este artículo parte de dicha investigación, de la que se publica un resumen digital al año siguiente (Vizcarra, 2015), pero sin embargo todo el material que proviene de los grupos de discusión es inédito, pues el análisis se realiza con posterioridad a esa fecha, y tiene el objetivo de profundizar en el concepto estudiado y en la percepción de dicho concepto por parte de la comunidad educativa. Este estudio de caso recoge las opiniones aportadas por las personas protagonistas del sistema educativo, que a través de cuestionarios y grupos de discusión ponen voz a la situación que vive la coeducación en sus centros escolares.

\subsection{Diseño}

Se diseñaron dos cuestionarios de respuesta abierta, uno dirigido al profesorado y el otro a las direcciones de los centros. A partir de la información recogida se profundizó en determinadas cuestiones a través de 7 grupos de discusión realizado con el profesorado, las familias y el alumnado, en los que se pudo reflexionar y compartir desde un planteamiento comunicativo y dialógico (Aubert, García y Racionero, 2009).

Entre las temáticas sobre las que profundizar destaca la conceptualización de la coeducación que es el punto central de este artículo, aspecto que hemos querido destacar por la importancia del tema a tratar. En los grupos de discusión se preguntó qué entendía la comunidad educativa por coeducación; cómo dibujaban la escuela coeducadora, qué características tenía esta noción compartida desde hace varias décadas, y si ha evolucionado su significado o si siempre se ha entendido de igual manera.

El estudio se ha realizado desde un enfoque cualitativo e interpretativo, ya que cobran importancia las interacciones con las personas participantes en la investigación con el fin de acercarse a la realidad (Monarca, 2015). La investigación cualitativa es de gran ayuda en lo que se refiere a relaciones sociales y a la pluralidad de mundos vitales porque proporciona una sensibilidad para el estudio empírico de los problemas (Flick, 2004, p. 15). Que los cuestionarios fueran abiertos aportó riqueza a la información recogida, ya que se pudieron desvelar qué significados otorgaba cada participante a los diferentes constructos culturales (Goetz y Lecompte, 1988), aportando también concreción a los detalles y a las diferentes interpretaciones aportadas sobre el tema trabajado (Bisquerra, 2004).

Además, con los grupos de discusión, se ha podido investigar sobre las características y dinámicas grupales como fuerzas constitutivas significativas en la construcción de cada significado y en la práctica de la vida social, porque exploran la naturaleza y los efectos del discurso social en desarrollo y facilitan la exploración de las memorias colectivas y las reservas compartidas de conocimiento (Denzin y Lincoln, 2015, p. 523).

\subsection{Participantes e instrumentos}

Se remitieron dos cuestionarios diferenciados, uno para el profesorado y otro para las direcciones de los centros. Respondieron 51 personas de equipos directivos, 24 mujeres (47\%), 25 hombres (49\%) y 2 que no concretaron su sexo-género (4\%). En cuanto al profesorado, contestaron 419 personas de las cuales 277 mujeres (66,1\%), 120 hombres $(28,6 \%$ y 22 que no lo especificaron (5\%). El número total de cuestionarios recibidos fueron 470. La edad media de quienes han respondido es de 
42,46 años y la media de años de experiencia docente es de 17,05 años. De las opiniones vertidas en los cuestionarios surgieron los grupos de discusión.

Los grupos de discusión contaron con la participación total de 36 personas: 10 en los grupos de discusión de profesorado y equipos directivos de los centros, 9 personas representantes de las familias y 17 estudiantes de secundaria. Los grupos de discusión fueron 7 en total: 2 de los grupos fueron con profesorado y equipos directivos, 2 con familias y 3 con alumnado. En ellos participan 14 mujeres y 2 hombres por el colectivo familias, 11 mujeres y 3 hombres por el colectivo de personal docente y 15 mujeres y 6 hombres por el colectivo estudiantes (el 85,5\% eran mujeres).

\subsection{Procedimiento general}

Para la recogida de datos de los cuestionarios se contó con la colaboración del personal del Departamento de Educación del Ayuntamiento de Vitoria-Gasteiz que hizo de intermediario entre el equipo de investigación de la Facultad de Educación y Deporte (UPV/EHU) y los centros escolares, y, por otro lado, los grupos de discusión también fueron convocados desde el propio Departamento de Educación.

Los cuestionarios creados ad hoc para la investigación permitieron descubrir qué significados otorgan las personas participantes a los diferentes constructos (Goetz y Lecompte, 1988). Tras analizar las respuestas recogidas y partiendo de las categorías emergentes, se elaboró el guion de los grupos de discusión que permitió obtener información acerca de lo que las personas piensan o sienten, sus percepciones, sentimientos y actitudes (Krueger, 1991).

El propósito del cuestionario era descubrir cómo se entiende el papel de los centros escolares en el camino hacia la coeducación, la percepción que se tiene en relación a su formación y las diferentes actitudes, sensibilidades y propuestas que aparecen en torno a este tema.

Mientras que con los grupos de discusión se desea comprender la realidad escolar en su conjunto, teniendo en cuenta a otras audiencias escolares que opinan sobre la coeducación para saber cómo entienden la escuela coeducadora, y qué características tiene.

\subsection{Procedimiento de análisis de la información}

Para analizar la información recogida en los cuestionarios y en los grupos de discusión se realizó un análisis semántico de contenido (Vallés, 2003). Con ese fin, se clasificaron temáticamente las distintas ideas expresadas en dichos grupos. En función de las interrogantes surgidas en los cuestionarios se elaboró el guion de preguntas que orientó las discusiones grupales. La construcción de ambos instrumentos, a su vez, estaba apoyada en la revisión teórica previa, y fue el punto de partida, en el que se incluyeron aquellos aspectos emergentes que surgieron de las aportaciones de cada participante en el cuestionario.

En ambos instrumentos se les preguntó acerca de la definición y el significado que otorgan al concepto coeducación y al modo en que se aplicaba en la práctica educativa. A continuación, se eligieron las voces de las personas participantes, bien por su claridad y su relevancia o bien porque presentaban matices específicos relacionados con la categoría trabajada.

La información recogida en las transcripciones de los grupos de discusión y la información recogida en los cuestionarios fue analizada mediante el software NVivo11.

Para realizar el análisis de la información recogida, se ha construido un sistema categorial (ver tabla 1) que ha sido construida siguiendo criterios inductivo-deductivos. Es decir, que inicialmente se apoya en la revisión teórica realizada, pero también recoge diferentes aspectos emergentes que surgen de las aportaciones de cada participante.

El interés de la investigación se centraba en conocer el significado que las personas de los centros otorgan a la coeducación.

Para identificar la procedencia de cada participante se ha confeccionado un código. El primer código binario es el del centro educativo (01... 30); el segundo código es el número de cada cuestionario por orden de aparición (01...45); en el siguiente código aparece el nivel educativo (Educación Infantil EI, Educación Primaria EP, Educación Secundaria ES); a continuación, el sexo-género, si es hombre H o si es mujer M. Los grupos de discusión (GD 1 a 7) llevan también el colectivo participante: familias (Fam), profesorado (Prof), alumnado (Alum). 
Los grupos de discusión han sido dinamizados por una persona del equipo de investigación y mientras otras dos personas tomaban notas de campo. Además, todas las sesiones han sido grabadas para su posterior transcripción y análisis.

Tabla 1.

Sistema categorial

\begin{tabular}{ll}
\hline Dimensiones & Indicadores \\
\hline $\begin{array}{l}\text { Qué entienden por coeduca- } \\
\text { ción (Recogida de diferentes visio- }\end{array}$ & Compartir espacio, estudiar juntos \\
nes y perspectivas sobre el término & Igualdad \\
en cuestión. Definiciones varias & Respeto a las diferencias \\
del concepto de coeducación) & Desarrollo personal integral \\
& Visibilización de lo femenino \\
& Revisión de la masculinidad hegemónica \\
& Ligadas a otros ámbitos (paz, convivencia) \\
\hline Trabajar la coeducación (Plas- & De quién es la responsabilidad \\
mación de la coeducación en prác- & Realizar sensibilización \\
ticas y reparto de tareas para su & Profesorado como modelo \\
consecución real y efectiva) & Transversalidad y trabajo en valores \\
& Necesidad de superar la brecha de género \\
& Desconocimiento \\
\hline
\end{tabular}

Fuente: elaboración propia

\section{Resultados}

En el momento en que el profesorado comienza a hablar sobre qué es lo que entienden por coeducación, se recogen diferentes conceptualizaciones que varían mucho en función de quien responde.

Casi el $90 \%$ de las voces recogidas identifican la coeducación con la educación mixta, es decir, la que se fija en el aprendizaje dirigido a grupos mixtos, a niñas y a niños en la misma aula, y casi todas estas respuestas son recogidas de los cuestionarios de Secundaria. Se menciona la educación conjunta de niñas y niños, la educación en el mismo espacio y de la misma manera, por lo que relacionan el término con la interpretación de escuela mixta, aunque se menciona que debe darse en un espacio no exclusivo, y con una educación integral.

«Educar a la gente, pero juntos, los unos con los otros» (1509 ES H)

«Clases mixtas e impartir valores, normas, estilos de vida, formación sin discriminar, todos pueden compartir las mismas tareas; no hay tareas, deportes, trabajos exclusivos» (2405 ES H)

«La educación integral de hombres y mujeres en el mismo espacio educativo» (2501 ES H)

La interpretación más compartida es la de la identificación con la igualdad. Casi todas las personas entrevistadas se refieren solamente a la igualdad, o también mencionan que supone la superación de las desigualdades existentes y con el despliegue de estrategias que ayuden en esta superación, así casi la mitad de las respuestas recogidas $(48 \%)$ tiene relación con algún aspecto que va en esta dirección.

«La educación que nos enseña que, aunque seamos de diferentes géneros, tenemos los mismos derechos» (0703 EI M)

«Educar en igualdad de oportunidades realmente, teniendo en cuenta estrategias para superar la situación de desequilibrio actual» (0206 EI H)

«Educar con los mismos valores a los niños de diferentes sexos, sin discriminación» (0501 EP M)

«Es educar a todos por igual sin diferencias de sexo, raza, economía, etc.» (2403 ES M)

En cuanto al respeto a las diferencias, mencionan a superación de roles y estereotipos de género heteronormativos. En este sentido las respuestas mayoritarias son las recibidas del profesorado de educación primaria (60 \%), mencionan el respeto, una educación basada en derechos y deberes, romper con los estereotipos de género. 
«Una educación a favor de romper los estereotipos sexistas» (2818 EI M)

«La igualdad de posibilidades, que no la uniformidad, y el respeto entre todos independientemente del sexo, raza y creencias» (1101 EP M)

«La educación basada en la igualdad de derechos y deberes que no discrimina por razón de sexo u orientación sexual y huye de roles sexistas u homófobos» (2611 ES M)

Una versión más actualizada del término la encontramos entre las personas que describen la coeducación como desarrollo personal por una parte y educación integral por otra. Uniendo de esta manera la educación con la perspectiva de género. Nos hablan del concepto de persona como una superación de la visión dicotómica que divide a la población en dos categorías excluyentes: hombres y mujeres, y nos hablan de la felicidad y el desarrollo de la individualidad, conceptos todos ellos bastante transformadores.

«Educar al alumnado sin hacer distinciones por sexo, descartando los roles de hombres y mujeres, y teniendo en cuenta a las personas en su totalidad» (2132 EP M)

«Capacidad de desarrollar en cada alumno/a una identidad personal que le haga feliz» (2407 ES H)

«Educación en igualdad, sin sesgos sexistas, que permita el desarrollo de la individualidad» (2410 ES H)

Aparece un único comentario que hace referencia a la incorporación de los saberes femeninos al currículo y la valoración positiva y visibilización del trabajo de las mujeres, de su aportación a la historia. Es llamativo que tan solo una de las respuestas apunte en esta dirección.

«Tratar por igual a chicas y chicos, y mencionar la igualdad en nuestras asignaturas: el papel de la mujer en la historia, el trabajo realizado por las escritoras...» (1835 ES H)

También en los grupos de discusión hay solamente una referencia relacionada con la revisión de la masculinidad hegemónica, y esta aparece unida al empoderamiento de las mujeres y a la necesidad de que los hombres también hagan su camino para superar el sistema sexo-género vigente.

\begin{abstract}
«Si yo incluso con mi hija (...) me encuentro perdida, porque yo he luchado por un tema que no sé cómo tengo que actuar ahora, con todo esto de los chicos y de las citas por internet, yo veo que en general son ellas las que llevan la voz cantante, les hemos dicho venga al poder, y nos encontramos con unas chicas que hacen mogollón de cosas (...) y ahora los que me preocupan son los chicos, que son los que no saben qué hacer. Igual, lo de la coeducación deberíamos también replantearlo para ayudar a que los chicos se recoloquen y encuentren su sitio. (...) parece que nosotras hemos tenido muchos motivos por los que luchar y reivindicar y que los chicos se encuentran perdidos» (140319 GDfam M)
\end{abstract}

La valoración de la diversidad también es mencionada una sola vez, además, de una manera muy vaga, sin embargo es destacable que hable de las diferencias como algo enriquecedor.

«Teniendo en cuenta las diferencias entre los sexos y partiendo de allí, es la educación que promueve la dignidad y la igualdad de derechos de chicos y chicas. Y la que ayuda a los dos sexos a respetarse y a estimarse, valorando y tomando como enriquecedoras las diferencias» (0828 EP H)

Existe quien enmarca la coeducación en la educación en valores o la educación para la paz y la convivencia, ambas tan en boga actualmente, y centradas en las emociones.

«La convivencia en paz de los seres humanos a diario, teniendo en cuenta los derechos y deberes de cada cual» (0821 EI M)

«La coeducación trata de que todos nuestros alumnos y alumnas puedan desarrollarse y mejorar sus capacidades en un entorno inclusivo, en igualdad y justicia» (0601 EP M)

«Educar en igualdad es que las chicas y los chicos no transmitan valores sexistas y que no acepten comportamientos inadecuados y los corrijan» (1830 ES M)

Aparte de las diferentes concepciones del término analizado, también se pueden encontrar reflexiones, provenientes especialmente de los grupos de discusión de las familias, reivindicando la necesidad de trabajar sobre la coeducación, ya que ven que es un término de largo recorrido, y mencionan que aún no se ha conseguido la igualdad, ni en los hechos, ni con la utilización de un lenguaje no sexista.

«Yo también pertenezco a una escuela de padres del instituto, y, por ejemplo, a la hora de elegir los temas, ese no salió, salieron los típicos, los de toda la vida, pero la coeducación no salió. Y la (...) psicóloga (...) nos dijo que había un repunte de la violencia, y nos animó a que no estaría mal que cogiéramos uno de esos temas, del sexismo, la violencia, o el maltrato (...) Los padres (...) el tema de la coeducación, es un tema que no nos lo habíamos planteado, (...) ni se me había pasado por la cabeza, pues eso, estás pensando en la adolescencia, la autoestima, no sé qué, no sé cuántos... pero coeducación...» (140319 GDfam M) 
«A mí me pasa como a mi hija que (...) decía: jjo! ¡qué pesada otra vez viene esta tía!, ¡qué pelma...! y venga que las mujeres somos iguales, pues jclaro! que no somos menos que somos iguales, pues claro... pero a estas alturas ¿me vas a venir a convencer que las mujeres somos iguales que los hombres?, pues, sí... Cuando nos vienen hablar de igualdad nos parecen las personas pelmas, pero mi entorno me dice que las cosas no están superadas» (140319 GDfam M)

También hay quien engloba el término y habla sobre la responsabilidad de la escuela y de la familia para trabajar conjuntamente, o la necesidad de formación que tiene el profesorado para poder hacerse cargo de este tema de una manera actualizada y adecuada.

«La educación en cooperación entre profesorado, familia y agentes sociales» (2014 EI M)

«En educación los diferentes agentes deben de ser partícipes» (2705 EP H)

«Tratamiento por igual a las personas, coherencia, formación interna, algo positivo... » (140305 GDprof M).

Se subraya la importancia en este tema de la concienciación y la sensibilización. Se habla del empoderamiento de las mujeres y de entrenar la mirada para saber distinguir las situaciones discriminatorias y de violencia simbólica de las que no lo son. Mencionan que a veces las situaciones de violencia las tenemos delante y no las vemos.

«Dar a las mujeres el lugar que perdieron, y tomar conciencia con mujeres y con hombres también, para conseguir derechos, porque nadie es menos que nadie» (0906 EI M)

«Al final la coeducación es saber qué tenemos que mirar, a dónde y cómo mirar, por lo que hay que comenzar formando la mirada, y luego saber qué hacer con ello. Sensibilizar para saber qué está pasando y qué hay que hacen» (1403051 GDprof M)

Se han fijado en la referencialidad del profesorado, pues son modelos también en cuestiones de género, y que la presencia y trabajo de la coeducación puede ser un indicador de calidad del centro en cuestión.

«Teniendo en cuenta que chicas y chicos son diferentes, ayudarles a desarrollar su identidad, sus capacidades, y construir relaciones respetuosas. Siempre sin olvidarse que el profesorado es ejemplo de formas de relacionarse» (0829 EP M)

«El trabajo que hacemos en educación es por un lado un reto que nos pone la vida, y por otro un criterio de calidad del centro educativo» (1510 ES H)

Otra idea a tener en cuenta que ha aparecido es la de la transversalidad de este tema, y de la existencia o no de planes y protocolos relacionados con el tema.

«Es una competencia transversal que hay que tener en cuenta en cada momento» (0818 EP M)

«Transmisión de valores, está presente en todas las asignaturas» (3020 EP M)

«Marcamos pautas e itinerarios para saber qué hacer» (1403051 GDprof M)

Sin embargo, es asombroso que haya personas que no expresan no saber definir el término, y así

al menos, tres personas han afirmado que no conocen el tema, y que no saben cómo definirlo.

«No había oído hablar de ello antes» (0511 EP M)

«No lo sé, porque no lo había oído nunca. Sí conozco el tema, pero desconocía que se refirieran a él en esos términos» (3005 ES M)

«No sé» (1817 ES M).

En general podríamos decir que la mayoría de las personas participantes en esta investigación no ha sido capaz de definir de una manera clara y concisa el término coeducación.

\section{Discusión y conclusiones}

En el momento que se solicita tanto en los grupos de discusión como en los cuestionarios de respuesta abierta definir libremente el concepto, las respuestas se dirigen en diferentes direcciones, que se relacionan con la escuela mixta, con la igualdad, con la educación entre diferentes, con la educación basada en valores y con la importancia de cuidar la convivencia, coincidiendo con los postulados de Miribilia (2011), al entender la coeducación como un acompañamiento ligado a la educación emocional en toda su extensión.

Este estudio muestra que, a pesar de llevar 40 años trabajando la coeducación en educación aún hay personas que no conocen el tema, ni saben cómo tratarlo, o incluso que eluden la respuesta. La coeducación debería ser algo que va más allá de compartir un espacio común debería ser una educación basada en el respeto a las diferencias y sin discriminación, por lo que, coincidiendo con Ballarín 
(2001) se debería plantear hacer un momento de pausa para repensar qué se necesita hacer en el entorno educativo sobre este tema.

La coeducación entendida como el respeto entre diferentes y como superación de los roles y estereotipos de género es mencionada fundamentalmente por el profesorado de educación primaria, etapa en la que se menciona en más ocasiones que en el resto de las etapas, por ello, se ve la necesidad de realizar investigaciones en esta materia que hagan evidente una revisión de la planificación educativa (Moreno, 2013; Ríos, 2015; Yugueros, 2015).

Son las familias en los grupos de discusión quienes reivindican con más ahínco la necesidad de trabajar la coeducación y el uso no sexista del lenguaje por entender que la sociedad aún está muy lejos de un trato de igualdad en derechos y obligaciones para mujeres y para hombres. Ven que es un término de largo recorrido, y que aún no se ha conseguido la igualdad, ni en los hechos, ni con la utilización de un lenguaje no sexista. En este sentido se debería revisar el currículo evitando que los modelos reinantes fueran los androcéntricos, tal y como propone Moreno (2013). Sin embargo, también creen que ya se ha hecho bastante trabajo con las chicas, e incluso hay quien valora negativamente a las mujeres empoderadas. Recogiendo entre las familias la misma polémica que existe en la sociedad. Consideran que éste es el momento de empezar a trabajar con los chicos sobre su rol y sobre su situación social (Yugueros, 2015)

Por las respuestas recogidas en los grupos de discusión se puede concluir que el profesorado que participó puso el énfasis en la necesidad de un tratamiento igualitario, en la necesidad de formación, y en la importancia de analizar la situación de la escuela y hacer un diagnóstico para luego poner en marcha una campaña de sensibilización, con el objetivo de trabajar la mirada de la comunidad escolar en este tema. En este sentido, la mayor parte de las fuentes consultadas coinciden en este punto (Aristizabal, Ugalde y Alvarez-Uria, 2017; Heredero de Pedro, 2013; Moreno, 2013; Leal y Aranda, 2011).

El alumnado reivindica los mismos derechos y oportunidades en todos los ámbitos y la igualdad en la educación, teniendo en cuenta que se le debe proporcionar ayuda a quien la necesite, dentro de ese trato igual. Ha quedado claro que aún se entiende la coeducación como sinónimo de igualdad, con ciertos matices como respeto a las diferencias, igualdad en un marco de transmisión de valores no machistas, e igualdad por medio de una educación mixta. Por lo que se deja en un segundo plano una conceptualización unida a un enfoque más actual, en el que se da más importancia al marco del desarrollo integral; a la incorporación de los saberes femeninos para convertir la educación en universal y que deje así de ser algo androcéntrico. Se debe revisar la idea de masculinidad hegemónica existente y se debe cuestionar la construcción y el papel social de los hombres; y la coeducación en relación con la visibilización, des-estigmatización y valoración positiva de la diversidad (de los colectivos sociales discriminados y de los tabús existentes en la escuela y en la sociedad, enmarcando las identidades sociales y culturales en términos de interseccionalidad).Por todo esto, se debe profundizar en la conceptualización de ciertos términos -como la coeducación- y en la sensibilización hacia la temática de género y feminista, tal y como proponen Ríos (2015), Subirats y Tomé (2007) y Subirats, (2016). De esta forma se impulsarán estrategias que ayuden a identificar situaciones de discriminación, violencia, relaciones de poder y comportamientos sexistas o androcéntricos que todavía se dan en el día a día de la escuela del siglo XXI.

Podemos concluir con las declaraciones en prensa de María Solana, cuando defiende el programa SKOLAE, nuevo plan de coeducación impulsado por el Gobierno de Navarra y realizado en red entre el movimiento feminista, la comunidad escolar y la administración. «No se educa en igualdad. Hay quien tiene proyectos concretos, profesorado que se ha formado en igualdad, o quien lleva toda la vida en el feminismo y son expertas en el tema. Pero hablamos de voluntarismo. Hasta hoy eso es lo que hemos tenido. Se realizó un diagnóstico profundo y debemos aceptar la situación» (Berasaluze, 2018, p. web). Se ve claramente que no todo el mundo quiere coeducar. Y para finalizar subraya que «Todo el mundo está a favor de la igualdad, en contra de la violencia, pero cuando ha de darse el paso decisivo, cuando llega el momento de pasar a la práctica, no hay acuerdo» (Berasaluze, 2018, p. web).

Este proyecto recién publicado merece su reconocimiento como proyecto referente y pionero, ya que tiene en cuenta el contexto actual y los posicionamientos ambivalentes, a favor, y en contra de la coeducación, para ser conscientes de las resistencias con las que se topa todavía en el día de hoy, cuando tanto las instituciones como la calle exigen un cambio ante el sexismo y la violencia que acarrea.

Por todo ello, de cara a la prospección de la investigación, los retos de la escuela coeducadora para el futuro, después de dos huelgas feministas en el 8 de marzo de 2018 y 2019, son los siguientes: 
1) Finalizar el diseño de planes de coeducación y comenzar a aplicarlos, es decir, pasar del discurso escrito a la comprensión de sus significados y su alcance y a las buenas prácticas diarias.

2) La participación de toda la comunidad escolar, y no sólo de las profesoras feministas, y el reconocimiento a la labor del movimiento feminista, sea de la corriente que sea (de la igualdad, de la diferencia, de la diversidad).

3) Facilitar formación y recursos para tratar temas, personal y colectivamente, como las relaciones de poder, las violencias y discriminaciones, o la interseccionalidad de las opresiones (género, sexualidad, raza-etnia, clase, lenguaje, capacitismo,...).

\section{Agradecimientos}

Está investigación se realizó a través de un convenio de investigación firmado entre el Departamento de Educación del Ayuntamiento de Vitoria-Gasteiz y la Universidad del País Vasco (UPV/EHU), y fue financiada con 12.000 euros. Nuestro agradecimiento a las personas y a los centros participantes y a aquellas personas del Departamento de Educación que nos facilitaron la labor en la recogida de la información.

\section{Referencias}

Araque, N. (2009). La educación en la Constitución de 1812: Antecedentes y consecuencias. Revista de la Facultad de Ciencias Sociales y Juridicas de Elche, 21(1), pp. 1-21.

Arenas, G. (2006). Triunfantes perdedoras. La vida de las niñas en la escuela. Barcelona: Graó.

Aristizabal, P., Ugalde, A. I., \& Alvarez-Uria, A. (2017). Yo elijo, tú eliges, ellas eligen. Orientación y toma de decisiones de las chicas en la escuela. Revista Española de Orientación y Psicopedagogía, 28(3), pp. 99-114. doi: https://doi.org/10.5944/reop.vol.28.num.3.2017.21621

Aubert, A., García, C., \& Racionero, S. (2009). El aprendizaje dialógico. Cultura y Educación, 21(2), pp. 129-139. doi: https://doi.org/10.1174/113564009788345826

Ballarín, P. (2001) La coeducación hoy. In N. Blanco (Coord.). Educar en femenino y en masculino. (pp.3140). Madrid: Akal.

Ballarín, P. (2006). La educación «propia del sexo». In C. Rodríguez (Coord.). Género y currículo. Aportaciones del género al estudio y práctica del currículo, (pp. 37-58). Madrid: Akal.

Berasaluze, G. (2018). Nahikoa da. (entrevista a María Solana). Berria, 2018/11/30. https://www.berria.eus/paperekoa/1913/004/001/2018-11-30/nahikoa_da.htm

Bisquerra, R. (2004). Metodología de la investigación educativa. Madrid: La Muralla.

Cabeza, A. (2006). Importancia de la coeducación en los centros educativos. Pedagogía magna, 8, pp. 39-45.

Calvo, A.; Susinos, T., \& García Lastra, M. (2011). El largo camino hacia la coeducación. Un análisis de las etapas de la educación de las mujeres a partir de tres relatos de vida escolar. Revista de Educación, 354, pp. 549-573

Codina, M. T. (2002). Rosa Sensat y los orígenes de los movimientos de renovación pedagógica. Historia de la educación, 21, pp. 91-104.

Denzin, N. K., \& Lincoln, Y. S. (Coords.). (2015). Manual de investigación cualitativa. Métodos de recolección $y$ análisis de datos. Barcelona: Gedisa. 
Emakunde. (2003). NAHIKO programa de coeducación para Educación Primaria (programa). Vitoria-Gasteiz: Gobierno Vasco. http://www.emakunde.euskadi.eus/contenidos/informacion/proyecto_nahiko_intro/es_def/adjuntos/nahiko.cas.pdf

Emakunde. (2003). NAHIKO programa de coeducación (página web). http://www.emakunde.euskadi.eus/nahiko/-/informacion/nahiko/

Flick, U. (2004). Introducción a la investigación cualitativa. Madrid: Morata.

Gobierno de Navarra. (2017). SKOLAE programa de coeducación (programa). Pamplona: Gobierno de Navarra. http://consejoescolar.educacion.navarra.es/web1/2017/09/08/skolae-creciendo-enigualdad/

Gobierno de Navarra. (2017). SKOLAE programa de coeducación (página web). http:/ / consejoescolar.educacion.navarra.es/web1/2017/09/08/skolae-creciendo-en-igualdad/

Gobierno Vasco (2013). Plan directorpara la Coeducación y la prevención de la violencia de género en el sistema educativo. http://www.euskadi.eus/contenidos/informacion/dig_publicaciones_innovacion/es_conviven/adjuntos/coeducacion/920006c_Pub_EJ_hezkidetza_plana_c.pdf

Goetz,J. P., \& Lecompte, M. (1988). Etnografía y diseño cualitativo en investigación educativa. Madrid: Morata.

Heredero de Pedro, M. C. (2013). El aprendizaje del género: reproducción y transformación de las relaciones de género en el discurso educativo. (Tesis doctoral) Universidad Complutense de Madrid.

Ianni, V. (2008). Guerra y revolución en España. México D.F: Ocean Sur.

Instituto de la Mujer (2008). Guía de coeducación. Sintesis sobre la Educación para la igualdad de oportunidades entre mujeres y hombres. Madrid: Ministerio de Igualdad. http://www.inmujer.gob.es/observatorios/observIgualdad/estudiosInformes/docs/009-guia.pdf

Krueger, R. A. (1991). El grupo de discusión. Guía práctica para la investigación aplicada. Madrid: Pirámide.

Leal, D., \& Arconada, M. A. (2011). Convivir en igualdad. Prevención de violencia masculina hacia las mujeres en todas las etapas educativas. Madrid: UDED.

Ley 1/1990 de 3 de octubre, Orgánica de Ordenación General del Sistema Educativo. Ministerio de Educación (LOGSE).

La Ley Orgánica 2/2006, de 3 de mayo, de Educación. Ministerio de Educación (LOE)

Ley Orgánica 8/2013, de 9 de diciembre, para la mejora de la calidad Educativa, del 23 de diciembre de $2013, \mathrm{BOE}, \mathrm{n}^{\circ} 295$

Martínez-Gorroño, M. E., \& Hernández-Álvarez, J. L. (2014). La institución libre de enseñanza y Pierre de Coubertin: la educación física para una formación en libertad. Revista Internacional de Medicina y Ciencias de la Actividad Física y el Deporte, 14, pp. 243-263.

Mirabilia, P. (2011). La coeducación en la escuela del siglo XXI. Madrid: Catarata.

Monarca, H. (2015). Políticas, prácticas y trayectorias escolares. Dilemas y tensiones en los procesos de inclusión. Perfiles educativos, 37(147), pp. 14-27.

Moreno, M. A. (2013). Queremos coeducar. Avilés: Centro de Profesorado y recursos de Avilés-Occidente.

Pujadas, X. (2009). Mujeres y deporte. Una larga carrera hacia la libertad. Mujer, 1, pp. 13-18. 
Riley, K. L. (2010). Fair and Tender Ladies versus Jim Crow: The Politics of Co-Education. American Educational History Journal, 37(2), pp. 407-417.

Ríos, O. (2015). Nuevas masculinidades y educación liberadora. Intangible Capital, 11(3), pp. 485-507. doi: http://dx.doi.org/10.3926/ic.654

Romero Rodríguez, S., \& Lugo Muñoz, M. (2014). Diagnóstico de las actitudes hacia la igualdady la coeducación. La perspectiva del profesorado de institutos de educación secundaria. $I+G$ 2014. Aportaciones a la Investigación sobre Mujeres y Género. Madrid: MINECO. https://idus.us.es/xmlui/bitstream/handle/11441/41074/Pages $\% 20$ from $\% 20$ Investigacion_Genero_14-2-13.pdf?sequence $=1$

Salas, B. (2003). El modelo coeducativo de la pentacidad. Aula de encuentro: Revista de investigación y comunicación de experiencias educativas, 7, pp. 147-153.

Suberviola, I. (2012). Coeducación: un derecho y un deber del profesorado. Revista de Enseñanza Interuniversitaria para la Formación del Profesorado, 15(3), pp. 59-67.

Subirats, M. (1994). Conquistar la igualdad: la coeducación hoy. Revista Iberoamericana de Educación, 6, pp. 49-78.

Subirats, M. (2016). De los dispositivos selectivos en la educación: el caso del sexismo. Revista de Sociología de la Educación, 9(1), pp. 22-36.

Subirats, M. \& Tomé, A. (2007). Balones fuera. Reconstruir los espacios desde la coeducación. Barcelona: Octaedro.

Tomé, A. (2001). La construcción de las identidades masculinas y femeninas en la escuela. In N. Blanco (Ed.). Educar en femenino y en masculino (pp. 87-98). Madrid: Akal.

Yugueros, A.J. (2015). La coeducación en la escuela como modelo de socialización. Ehquidad International Welfare Policies and Social Work. Journal, 4, pp. 61-70. doi: 10.15257/ehquidad.2015.0009.

Vallés, M. S. (2003). Técnicas cualitativas de investigación social. Madrid: Síntesis.

Vizcarra, María Teresa. (2015). Estudio-diagnóstico sobre coeducación en centros educativos de VitoriaGasteiz. (0 a 18 años). Vitoria-Gasteiz: Departamento de Educación del ayuntamiento. https://www.vitoria-gasteiz.org/docs/wb021/contenidosEstaticos/adjuntos/es/80/50/58050.pdf 